\title{
Apprentissage en binôme collaboratif dans l'enseignement pratique de la formation initiale des pédicures-podologues : une enquête de terrain
}

\section{A survey of peer learning in the initial training of French podiatrists}

\author{
Karine DELAHAYE-MULLER ${ }^{1, *}$, Yann LE FAOU ${ }^{2}$, et Gérard CHALES ${ }^{3}$
}

${ }^{1}$ Pédicure-Podologue DE, Formatrice au collège des hautes études en médecine, Ex-vacataire à l'Institut de formation en pédicurie-podologie, ergothérapie et masso-kinésithérapie de Rennes, Rennes, France

2 Coordonnateur recherche à l'Institut de formation en pédicurie-podologie, ergothérapie et masso-kinésithérapie de Rennes, Doctorant en sciences de l'éducation, Université de Rennes 2, CREAD EA 3875, Rennes, France

${ }^{3}$ Rhumatologue, Université de Rennes 1, Faculté de médecine, 35043 Rennes, France

\begin{abstract}
Manuscrit soumis à la rédaction le 18 avril 2017 ; commentaires éditoriaux formulés aux auteurs le 11 mars, le 17 octobre et le 14 novembre 2018 ; accepté pour publication le 15 novembre 2018

Résumé - Contexte : En France, les étudiants en pédicurie podologie se forment en binôme de pairs sur les compétences techniques lors des stages pratiques dans les instituts de formation. But: Évaluer le vécu des étudiants lors de cette formation collaborative en binômes : degré de satisfaction, vécu du dispositif et évolution souhaitée de la formation. Méthode: Un questionnaire en ligne combinant dix questions fermées et une question ouverte a été conçu. Il a été diffusé, d'une part, en présentiel et, d'autre part, via le réseau social Facebook pour les jeunes diplômés. Résultats : Le pourcentage d'opinions positives pour l'ensemble des questions fermées était supérieur à $50 \%$. La qualité du stage était jugée satisfaisante et très satisfaisante. Les points forts identifiés par les étudiants dans les questions fermées concernaient l'importance du travail en binôme dans leur apprentissage au pôle de soin, l'absence de gêne pour poser des questions, la capacité d'expliquer simplement, d'une part, les liens entre pathologies, morphologies et environnement, d'autre part, la démarche thérapeutique. De manière générale, les étudiants notent qu'ils sont à l'aise avec leur binôme. Conclusion: Ce dispositif pédagogique semble être apprécié des étudiants. Toutefois, l'organisation mériterait d'être mieux institutionnalisée et reconnue, afin que les étudiants le voient d'abord comme une chance pour leur formation de clinicien et non comme une obligation imposée par l'institution. Pour reconnaître les compétences de tuteur des étudiants, une formation au tutorat pourrait être proposée aux étudiants dès la première année de formation.
\end{abstract}

Mots clés : pédicurie-podologie, formation initiale, stage clinique, formation entre pairs, tutorat

\begin{abstract}
Context: In France, podiatry students work in pairs (near-peer: two students from different cohorts) during clinical practice training sessions organized by the podiatry teaching institute. Aim: To evaluate the collaborative work from a peer-perspective (degree of satisfaction, scheme experience and anticipated training progress). Method: An online questionnaire that included ten closed and one open question was designed. It was first handed out in class and then through Facebook to young graduates. Results: The percentage of positive opinions for all closed questions was over $50 \%$. The quality of practice training sessions was considered adequate or very adequate. The strong points identified by students on closed questions related to the significance of peer-work learning in the care center, the lack of discomfort in asking questions, the ability to explain the links between diseases, morphologies and environment on the one hand and the therapeutic approach on the other. On the whole, students mention that they were satisfied with the pairing. Conclusion: Students seem to appreciate the educational scheme. However, its organization would deserve to be better institutionalized and recognized in order for students to see it as an opportunity rather than an obligation in their training as professional practitioners. For tutor competencies to be recognized, a tutoring course could be recommended to students as early as the first year of training.
\end{abstract}

Keywords: podiatry, peer work/learning, initial training, evaluation

\footnotetext{
*Correspondance et offprints: Karine DELAHAYE-MULLER, 45 avenue de la Gare, 35360 Montauban de Bretagne, France. Mailto: cabinetdelahayemuller@gmail.com.
} 


\section{Introduction}

L'apprentissage par les pairs a pour but le développement des connaissances et des habiletés par l'entraide et le soutien entre personnes de même statut ou entre collègues jumelés qui ne sont pas des enseignants professionnels [1]. Ce mode d'apprentissage historique a été de développé dans les pays anglo-saxons dans les années 1980 en formation générale et introduit ultérieurement de façon informelle dans plusieurs dispositifs de formation en santé: médecins [2], infirmières [3], kinésithérapeutes, dentistes, psychologues.

La littérature en sciences de l'éducation a démontré l'intérêt de l'apprentissage par les pairs et ses bénéfices cognitifs, pédagogiques, psychologiques, sociaux et économiques [1]. Plus récemment, a émergé un intérêt pour une approche plus formelle, organisée et planifiée, de l'apprentissage par les pairs chez les étudiants.

\section{Contexte et cadre conceptuel}

Dans le contexte français, la formation des pédicurespodologues, comme celle de plusieurs autres professionnels de la santé, est planifiée dans le cadre d'une «approche par compétences [4]». Un référentiel d'activités, un référentiel de compétences et un référentiel de formation pour le métier de pédicure-podologue diplômé d'État ont ainsi été publiés en annexes de l'arrêté du 5 juillet 2012 relatif au diplôme d'État de pédicure-podologue [5]. Les recommandations concernant les dispositifs curriculaires et pédagogiques à mettre en œuvre mettent en avant le principe d'enseignement et d'apprentissage contextualisé et la posture réflexive, en souhaitant favoriser l'autonomie de l'étudiant et le transfert des apprentissages, en lien avec les cadres reconnus pour la formation des professionnels de la santé $[4,6]$. Le référentiel de formation prévoit une alternance des temps de formation entre les enseignements théoriques et les apprentissages pratiques, dans le cadre d'un total de 5400 heures de formation, dont 1170 heures de formation clinique (stage extrahospitalier et hospitalier), réparties sur trois années.

Certains instituts de formation en pédicurie-podologie accueillent un centre clinique où les étudiants pratiquent des soins sur des patients dès la fin du premier trimestre de la formation. Les patients sont encadrés par un binôme (étudiant $1^{\text {re }}$ année - P1-/étudiant de $3^{\mathrm{e}}$ année-P3). Cet apprentissage de la collaboration à l'institut de formation est proposé aux étudiants avant tout pour des raisons pédagogiques (espace de co-formation collaborative) mais aussi logistiques (faire travailler plusieurs groupes d'étudiants de niveaux différents sur un même temps et un même lieu de stage). Le roulement rapide des étudiants sur l'année empêche une régularité dans le binôme et donc dans la relation entre les pairs.

Les relations entre pairs visant l'accompagnement ou la remédiation peuvent prendre diverses formes, qui ont été définies par Paul [7]. On parle parfois de compagnonnage (transmetteur de «savoir-faire», de «pratique gestuelle», voire de règles et de valeurs professionnelles et déontologiques), mais aussi de mentorat (modèle tant pour les qualités professionnelles que pour les qualités morales que l'accompagné attribue au mentor) ou encore de tutorat (organisateur du parcours de l'étudiant en formation dans un cadre formel, voire institutionnalisé) [8]. Au-delà de la simple maîtrise du savoir, ces trois postures d'accompagnement mettent en jeu les notions d'habiletés (savoir-faire) et d'attitudes (savoir-être) décrites selon trois domaines d'objectifs (cognitif, psychomoteur et psychoaffectif) dans plusieurs taxonomies telles que, par exemple et respectivement, celle de Bloom, de Harrow et de Kratwhol [9].

Dans le cadre d'un binôme d'étudiants de niveaux différents, celui de l'année supérieure est considéré comme «aidant, accompagnant ou expert». Il joue le rôle de superviseur «de l'aidé ou du novice » : il s'agit ici de pairs de proximité (near-peer), «l'expert » ayant plus d'expérience et plus de connaissances que son «novice». La posture d'accompagnement la plus proche du travail collaboratif en formation initiale des pédicures-podologues serait donc ici celle du compagnonnage [7].

À notre connaissance, peu d'études concernent l'évaluation de l'apprentissage par les pairs en pédicuriepodologie, en dehors d'une approche sociologique des étudiants du binôme. En revanche, des travaux montrent que l'apprentissage par les pairs (jumelage entre étudiants de première année et de deuxième ou troisième années) était considéré comme une solution intéressante pour les stages en soins infirmiers, tant au Québec (où le jumelage est perçu comme source de motivation et de satisfaction, avec une relation agréable entre les jumeaux [10]) qu'à Hong-Kong (où le jumelage collaboratif conduisait à une utilisation plus fréquente de stratégies de raisonnement de haut niveau (approche réflexive, esprit critique) et à un plus grand transfert des apprentissages [11]. Dans une étude expérimentale récente, le jumelage entre étudiantsinfirmiers de la même année (dernière année de formation initiale) entraînait une augmentation de niveau du sentiment de perception de compétence pour gérer les activités et les défis de la profession [3]. L'apprentissage par les pairs est aussi fréquemment utilisé en médecine où il peut prendre de multiples formes (compagnonnage, mentorat, tutorat, etc.) et où il est perçu comme bénéfique [2] à la fois pour les binômes accompagnant/enseignant, accompagné/apprenant [12] et pour l'institution. Ce dispositif permet de réduire la tâche d'enseignement des enseignants, grâce à un accompagnant 《expert » ayant des connaissances de base similaires à celles de l'accompagné «novice» (congruence). Du point de vue de l'institution, il favorise la création d'un environnement éducatif confortable et sûr, dans le but de fournir des modèles de rôle [13] pour les étudiants-juniors et de préparer les étudiants-séniors à leur futur rôle d'éducateur pour développer leur leadership. Indirectement, l'apprentissage par les pairs offre aux étudiants une motivation alternative (il mobilise la motivation intrinsèque), ainsi qu'une autre façon d'apprendre. L'apprentissage par les pairs permet dans un second temps de modifier la culture académique en invitant à considérer l'éducation comme une tâche 
essentielle dans les soins en santé, tout en pérennisant des programmes d'entraînement-formation dans un contexte de ressources limitées.

\section{Objectif}

Dans le cadre de notre expérience de l'apprentissage avec les pairs en formation initiale de pédicures-podologues, plusieurs questions ont émergé: comment les apprenants novices vivent-ils cet exercice? L'étudiant novice ou expert est-il à l'aise avec sa posture d'accompagnement? Comment évalue-t-il ce dispositif pédagogique? Que souhaiterait-il voir évoluer? Nous avons donc entrepris une enquête exploratoire visant à documenter la pertinence de ce type de dispositif pédagogique (travail en binôme), telle qu'elle était perçue par les étudiants en formation initiale de pédicurie-podologie.

\section{Méthodes}

\section{Populations étudiées}

La population cible était composée de deux groupes : 1) 128 étudiants issus de trois promotions (P1, P2 et P3) en formation pendant l'année universitaire 2015/2016 à l'Institut de formation en pédicurie-podologie de Rennes ; 2) 84 jeunes diplômés recrutés à partir de deux promotions issues du même établissement.

\section{Activités concernées}

Il s'agissait en particulier d'étudier les étudiants de première et troisième années travaillant en binômes en situation professionnelle, c'est-à-dire au cours de situation d'apprentissage de techniques professionnelles sur des patients dans le centre de soins de l'institut.

Chaque étudiant gère un poste de travail (unité, plateau technique...) organisé de son côté du fauteuil de soins (un étudiant par pied d'un même patient). D'un côté, l'apprenant «novice» observe, applique les conseils et propositions de son binôme; il peut poser librement ses questions et demander l'aide de son collègue d'année supérieure. De l'autre côté, l'étudiant «expert» mène l'interrogatoire et s'axe sur la relation patient. Il vulgarise ainsi les termes complexes pour son binôme novice, explique sa démarche et son raisonnement clinique. Parallèlement à ce binôme étudiant, il existe aussi un binôme de formateurs, l'un d'entre eux étant un formateur permanent issu de l'institut et l'autre, un formateur clinicien, vacataire exerçant une activité libérale. Les formateurs interviennent à tout moment sur demande des étudiants, sur l'observation et le diagnostic en pédicurie-podologie, ainsi que systématiquement en début et à la fin de chaque soin afin de valider la prise en charge du patient.

\section{Questionnaire d'enquête}

Un questionnaire d'enquête explorant le vécu [14] des étudiants par rapport au stage pratique à la clinique de soins, et tout particulièrement à l'apprentissage par binôme, a été administré en ligne, de façon anonyme, respectivement à partir de l'application Google Form pour les étudiants et via le réseau social Facebook pour les jeunes diplômés (en raison de la non-disponibilité de leur adresse de messagerie électronique).

Ce questionnaire était composé de trois sections. La première explorait le profil des personnes interrogées. La deuxième, composée de 10 questions sollicitant une réponse sur une échelle de Likert à cinq points, explorait le vécu des étudiants par rapport au dispositif pédagogique (promotion P1, P2, P3 et jeunes diplômés) concernant le travail en binômes. Cette rubrique comporte 10 questions fermées et une question rédactionnelle. Cela en fait une enquête mixte à la fois quantitative et qualitative. Le temps nécessaire pour le remplir est de moins de cinq minutes.

Les questions ont été élaborées en lien avec les compétences identifiées dans le référentiel. Quatre thèmes en ont été extraits pour construire les questions relatives à la démarche thérapeutique, la technicité professionnelle, le raisonnement clinique et la relation soignant-soigné. Les autres questions ont été élaborées à partir d'un questionnaire exploratoire, administré à 14 étudiants (sept en P1 et sept en P3). Six questions additionnelles ont pu ainsi être formulées.

Parmi les 10 questions, présentées dans le tableau I, neuf sollicitaient une réponse sur une échelle de Likert à cinq items (de «pas du tout d'accord» à «tout à fait d'accord») comportant une modalité médiane (3). Il s'agit de laisser le choix aux étudiants et non de les obliger à prendre parti. L'échelle d'attitude est une technique de mesure de l'intensité des opinions ou des réactions des individus sur un sujet donné portant sur des notions plus objectives (plus descriptives) comme les comportements [15]. La dernière question (Q10) sollicitait une réponse ouverte, sans limitation du nombre de mots.

\section{Analyse}

Les données quantitatives brutes ont été extraites et traitées dans un tableau Excel. Elles sont présentées sous forme de graphes en barres horizontales concernant le niveau de satisfaction des étudiants vis à vis de chaque domaine exploré (Figure 1); les valeurs ordinales générées par les réponses sur l'échelle de Likert ayant été traitées comme des valeurs d'intervalle [16] (Figure 2).

Les données qualitatives générées par la question 10 ont été traitées dans le cadre d'une analyse de contenu. Une première étape a visé la sélection des items (unité lexicale) puis le codage en étiquettes, selon une démarche inductive. Une deuxième étape a permis de regrouper des étiquettes, de proche en proche, sous une catégorie, en tenant compte du poids (comptage et prévalence) de chaque étiquette. Enfin, une troisième étape a consisté en une analyse thématique. 
Tableau I. Questionnaire administré aux participants de l'étude (les réponses étaient sollicités sur une échelle de Likert à 5 points de «pas du tout d'accord» à «tout à fait d'accord»).

\begin{tabular}{ll}
\hline Q1 & Le travail en binôme est un dispositif important dans mon apprentissage au pôle soin. \\
Q2 & Je me sens à l'aise avec mon binôme. \\
Q3 & Je sollicite mon binôme au cours de la séance. \\
Q4 & Je me sens gêné(e) de poser des questions à mon binôme devant le patient. \\
Q5 & Je me sens capable d'expliquer ma démarche thérapeutique à mon binôme. \\
Q6 & Mon binôme m'aide dans l'apprentissage de la technicité en pédicurie. \\
Q7 & Mon binôme m'aide dans le raisonnement clinique en pédicurie. \\
Q8 & Mon binôme m'aide dans la relation soignant/soigné. \\
Q9 & Je me sens capable de vulgariser (d'expliquer simplement) à mon binôme les liens entre pathologies, morphologies, \\
& environnement. \\
Q10 & Comment jugez-vous la qualité globale de ce stage pratique?
\end{tabular}

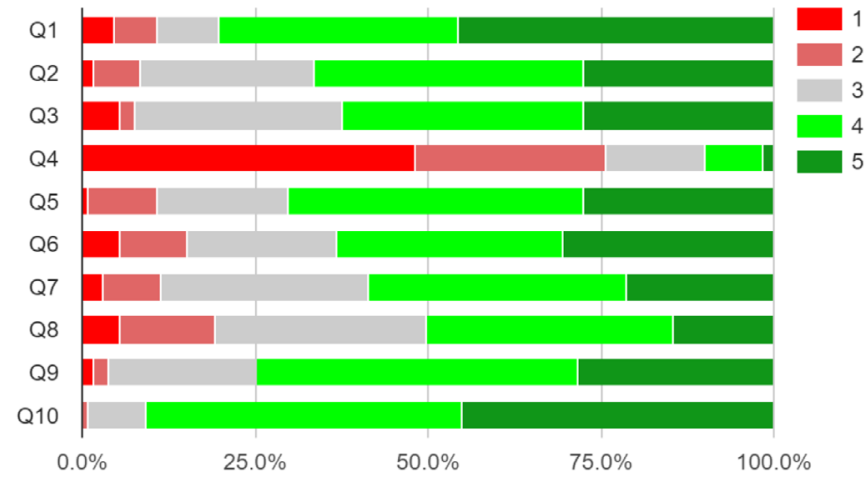

Fig. 1. Répartition des réponses des étudiants aux questions de l'enquête. Questions (Q) : 1: Le travail en binôme est un dispositif important dans mon apprentissage au pôle soin ; 2 : Je me sens à l'aise avec mon binôme; 3 : Je sollicite mon binôme au cours de la séance ; 4 :Je me sens gêné(e) de poser des questions à mon binôme devant le patient ; 5 : Je me sens capable d'expliquer ma démarche thérapeutique à mon binôme; 6 : Mon binôme m'aide dans l'apprentissage de la technicité en pédicurie; 7 : Mon binôme m'aide dans le raisonnement clinique en pédicurie ; 8: Mon binôme m'aide dans la relation soignant/soigné; 9 : Je me sens capable de vulgariser (d'expliquer simplement) à mon binôme les liens entre pathologies, morphologies, environnement ; 10 : Comment jugez-vous la qualité globale de ce stage pratique? Échelle de Likert à 5 ancrages (1: Totalement en désaccord à 5 : Totalement d'accord).

\section{Résultats}

Parmi les 212 personnes sollicitées, 131 ont répondu au questionnaire (taux de réponse $61,8 \%$ ) : 34 étudiants en P1, 30 étudiants en P2, 30 étudiants en P3 et 37 jeunes diplômés; 78 étaient des femmes et 53 des hommes; les trois-quarts étaient âgés de 20 à 24 ans.

Le pourcentage d'opinions positives (niveaux 4 et 5 de l'échelle de Likert) était supérieur à $50 \%$. Les points forts (opinion positive) identifiés par les étudiants concernaient l'importance du travail en binôme dans leur apprentissage au pôle de soin (Q1 : 80,6\%), l'absence de gêne pour poser des questions (Q4: 75,6\%), la capacité d'expliquer simplement d'une part les liens entre pathologies, morphologies et environnement (Q9: 74,8\%), d'autre part la démarche thérapeutique (Q5: 70,2\%), le fait de se sentir à l'aise avec son binôme (Q2: 66,4\%). En revanche, l'opinion était plus nuancée $(<2 / 3)$ concernant l'apprentissage des gestes techniques (Q6: 63,4\%), la sollicitation du binôme au cours de la séance (Q3: 62,3\%), l'aide au raisonnement clinique (Q7 : 58,8\%) et à la relation soignant/soigné (Q8 : 50,5\%).
Les moyennes arithmétiques des valeurs numériques correspondant aux réponses sur l'échelle de Likert sont indiquées sur la figure 2 .

Les données qualitatives sont présentées dans le tableau II. Plusieurs points positifs du travail entre pairs sont soulignés: 116 étudiants (sur les 131) mettent en exergue l'échange (45 sur 116), le partage d'expériences et de techniques (40), la mise en confiance/moins de stress (26), les conseils (15), l'évaluation par rapport à l'autre (11), la disponibilité (6), le compagnonnage (5) et la motivation (5). Certains points négatifs sont mentionnés: 108 étudiants (sur les 131) rapportent ainsi le manque de place/problème avec le matériel (48 sur 108), le caractère insuffisamment représentatif de la situation d'apprentissage par rapport à la vie professionnelle (27), la difficulté à acquérir de la vitesse (26), le manque d'autonomie (19), l'impression de ralentir l'autre (12), le risque de copier les erreurs (7), l'insuffisance de lien avec le patient (6), le manque d'affinité dans le binôme (5), la perte de temps pour expliquer au novice (5) et enfin le désaccord thérapeutique (3). 


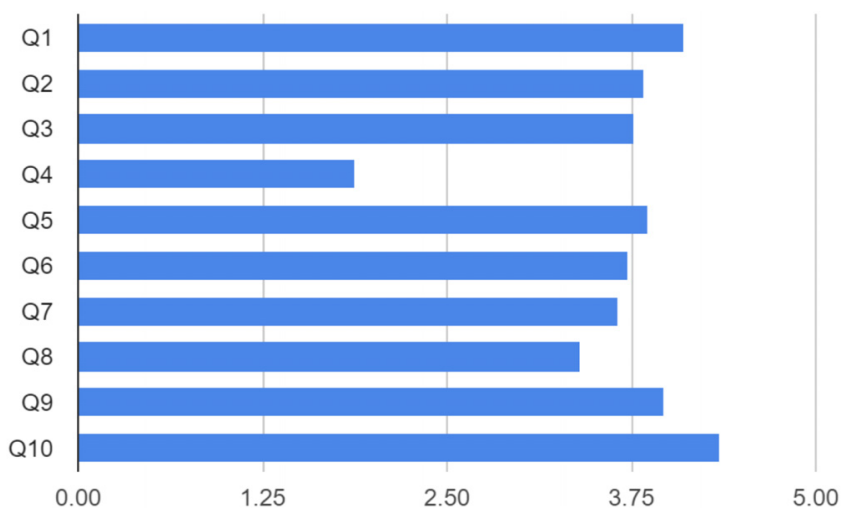

Fig. 2. Moyenne arithmétique des réponses au questionnaire de l'enquête sur l'échelle de Likert (tendance centrale des réponses sur le travail entre pairs). Questions (Q) : 1 : Le travail en binôme est un dispositif important dans mon apprentissage au pôle soin (moy. 4,11 ET 1,10) ; 2 : Je me sens à l'aise avec mon binôme (moy. 3,84, ET 0,96) ; 3 : Je sollicite mon binôme au cours de la séance (moy. 3,77 ET 1,05) ; 4 :Je me sens gêné(e) de poser des questions à mon binôme devant le patient (moy. 1,88 ET 1,05); 5 : Je me sens capable d'expliquer ma démarche thérapeutique à mon binôme (moy. 3,86 ET 0,96); 6: Mon binôme m'aide dans l'apprentissage de la technicité en pédicurie (moy. 3,73 ET 1,16) ; 7 : Mon binôme m’aide dans le raisonnement clinique en pédicurie (moy. 3,66 ET 1,01) ; 8 : Mon binôme m'aide dans la relation soignant/soigné (moy. 3,40 ET 1,07); 9: Je me sens capable de vulgariser (d'expliquer simplement) à mon binôme les liens entre pathologies, morphologies, environnement (moy. 3,98 ET 0,85); 10 : Comment jugez-vous la qualité globale de ce stage pratique? (moy. 4,35 ET 0,67). Échelle de Likert à 5 ancrages (1 : Totalement en désaccord à 5 : Totalement d'accord).

\section{Discussion}

\section{La relation entre pairs: un vecteur d'apprentissage}

D'une manière générale, l'indice de satisfaction des étudiants pour ce stage pratique est très important.

D'une part, les objectifs de la relation d'aide sont atteints : l'expert adopte une posture d'accueil mettant à l'aise son novice (Q2), de disponibilité (Q5, Q9) ou de technicité (Q6) ; le novice se sent à l'aise dans cette relation (Q2, Q3, Q4), ce qui réduit la distance entre novice et expert. Pour les étudiants interrogés, le travail en binôme est un dispositif favorisant l'apprentissage (Q1); il constitue un accompagnement nécessaire dans l'enseignement de la pratique professionnelle au regard de: la mobilisation de la technicité pédicurale (Q6), le raisonnement clinique (Q7), la relation soignant/soigné (Q8). De plus, il permet à l'étudiant d'expliquer à un autre sa démarche clinique avec un vocabulaire vulgarisé (appropriation et transmission des connaissances) (Q5, Q9).

\section{Des freins à l'apprentissage par les pairs}

Bien que le travail collaboratif soit admis comme étant un bon moyen de progresser en première et deuxième année, il est perçu comme un frein à partir de la troisième année, en particulier au dernier semestre de leur formation. De plus, les étudiants de P2 et P3 demandent également à travailler en «temps contraint », c'est-à-dire en imposant une limite de temps pour réaliser le soin afin que le temps imparti se rapproche le plus possible du temps d'une consultation classique en libéral. Ils sont 26 (sur 108 répondants) à trouver que le travail en binôme les empêche d'acquérir de la vitesse d'exécution dans la pratique de soin et 27 à penser que la pratique au centre de soins n'est pas assez représentative de la vie professionnelle. En stage pratique à l'institut, le temps «aux pieds » du patient est en moyenne de 75 min pour un pied, alors qu'en pratique libérale ce temps de soin n'excède pas les 35 minutes pour les deux pieds. Cette demande de travailler en temps contraint est aussi relayée par les jeunes diplômés qui ne se sentent pas compétents lors des premiers remplacements en libéral: lenteur pour certains ou manque d'autonomie dans le travail pour d'autres.

Parallèlement à cette demande d'autonomie en fin de cursus, définie comme «capacité d'agir par soi-même» [17], l'enquête montre une inadaptation du matériel de soins; par exemple, l'absence de jambières non-écartables sur de nombreux poste de travail ou encore le nombre insuffisant d'unités » en état de fonctionnement (panne eau/moteur). L'espace de travail devrait permettre à chacun d'exercer sans se gêner (séparation des espaces de travail). Sans cette précaution, le travail en binôme peut devenir contraignant pour les étudiants, ce qui implique donc d'améliorer les conditions organisationnelles des soins pour améliorer les conditions d'un apprentissage par pairs.

\section{Préconisations}

Le dispositif d'apprentissage par les pairs mériterait d'une part d'être privilégié, au regard de ses potentialités. Il mériterait d'autre part d'être mieux formalisé dans le référentiel de formation [8]. Si l'accent était mis sur une situation analogue à celle vécue en cabinet, entre un expert dans le rôle du soignant et un novice dans celui du malade, auquel on explique sa pathologie, la prise en charge nécessaire et son traitement, les étudiants de P3 ne le verraient plus comme un handicap ou une gêne mais comme un bénéfice. 
Tableau II. Points positifs et négatifs du travail entre pairs d'après les étudiants en prenant en compte la prévalence des notions contenues dans les réponses.

\begin{tabular}{|c|c|c|c|}
\hline Points positifs & 116 & Points négatifs & 108 \\
\hline Communication/échange/ question & 45 & Manque de place/gêne avec le matériel (jambières) & 48 \\
\hline Partage de l'expérience/des techniques & 40 & Pas représentatif de la vie professionnelle & 27 \\
\hline Etre mis en confiance /- de stress & 26 & Ne permet pas d'acquérir de la vitesse & 26 \\
\hline Conseils et entraide & 15 & Manque d'autonomie & 19 \\
\hline Évaluation par rapport à l'autre/états des lieux & 11 & L'impression de ralentir l'autre & 12 \\
\hline Disponibilité & 6 & Faire comme l'autre/copier les erreurs & 7 \\
\hline Compagnonnage & 5 & Pas d'intimité/d'implication avec le patient & 6 \\
\hline \multirow[t]{3}{*}{ Motivation/progression } & 5 & Manque d'affinité dans le binôme & 5 \\
\hline & & Perte de temps à expliquer au P1 & 5 \\
\hline & & Désaccords thérapeutiques & 3 \\
\hline
\end{tabular}

Par ailleurs, la moindre adhésion des étudiants de troisième année vis-à-vis du travail entre pairs pourrait être un indicateur d'une demande d'autonomie en fin de cursus. Ainsi, il serait intéressant, lors du dernier semestre, de permettre aux étudiants de troisième année de travailler plus souvent seul avec leur patient. En effet, la demande importante des étudiants d'être mis en temps contraint correspond à leur souhait de s'autoévaluer dans la gestion de leur temps et de leur stress sur un temps imparti. Nous pourrions envisager d'ajouter dans le scénario de formation une séquence où l'on tient compte de cette graduation de l'apprentissage, afin de renforcer la perception de compétence de l'étudiant.

\section{Limites de l'étude}

L'étude présente trois limites majeures:

- premièrement, concernant l'ensemble de l'étude, l'un des auteurs enseignait dans cet institut de formation au moment de cette recherche, ce qui a peut être induit un biais. Toutefois, il est limité par le fait que cette enquête a été effectuée dans le cadre d'un travail académique en pédagogie médicale, explicité aux enquêtés et non commandité par l'institut de formation;

- deuxièmement, l'échelle de Likert et le questionnement par affirmation posent deux problèmes. L'utilisation d'échelle de Likert à réponse numérique impaire implique une modalité centrale «3» (ni accord, ni désaccord), qui est difficile à analyser (réponse refuge pour celui ou celle qui ne sait quoi répondre). Les enquêtés peuvent être attirés par facilité ou manque de motivation ou encore hésiter dans une réponse, mais cette limite ne semble pas avoir influencé les données, car on ne compte que 274 réponses médianes sur 1309 réponses sollicitées sur l'ensemble du questionnaire. De plus, concernant l'affirmation des réponses, il peut être concevable que l'enquêté veuille faire plaisir à l'enquêteur (l'enquête ayant été administrée par le chercheur, à l'époque formatrice au centre de soins) et apporte un biais d'administration lors du recueil des données. Toutefois, ce risque est limité par le fait qu'il s'agit d'un questionnaire en ligne sans interaction directe avec le chercheur. Enfin, les scores de la figure 2 sont issus du calcul des moyennes des réponses obtenues sur l'échelle de Likert : les valeurs ordinales générées par les réponses sur les échelles de Likert sont donc traitées comme des valeurs d'intervalle, même s'il existe un débat dans la littérature sur la légitimité de cette façon de procéder [16];

- troisièmement, pour la question à réponse ouverte, l'extraction de l'information se fait par comptage de mots clés mais aussi de notions connexes, ce qui introduit un biais interprétatif dans les regroupements de catégories.

\section{Conclusion}

Lors des stages pratiques en formation initiale des pédicures podologues, les étudiants se forment et soignent les patients en binôme de pairs. L'enquête a mis en avant la satisfaction des apprenants concernant le dispositif de travail-formation entre pairs.

Le compagnonnage centré sur la transmission d'habiletés et d'attitudes (rapport soignant/soigné, comportement, identité et déontologie professionnelle) implique activement l'étudiant expert ainsi que l'étudiant novice dans l'incorporation des compétences de la profession. Néanmoins, en dernière année, les étudiants montrent un désengagement dans le travail-formation entre pairs, en lien avec une demande d'autonomie et un souci de rapidité dans l'exécution des gestes professionnels.

Institutionnaliser et scénariser l'apprentissage entre pairs apparaît essentiel afin de formaliser le rôle des étudiants et des formateurs mais surtout de reconnaître l'étudiant «tuteur» dans son rôle de formateur. Les résultats de notre enquête suggèrent l'importance d'un projet pédagogique intégrant une formation des étudiants aux situations de travail-formation en binôme, dès leur première année de présence à l'institut. En complément de la formation à cette méthode pédagogique, l'ergonomie des postes de travail doit être assurée afin que l'aspect 
technique du soin ne soit pas un frein au dispositif pédagogique par les pairs mis en place en formation initiale.

\section{Contributions}

Karine Delahaye-Muller a conçu le protocole de recherche, effectué le recueil des données, l'interprétation des résultats, l'analyse statistique et l'écriture du manuscrit. Yann Le Faou et Gérard Chalès ont contribué à l'interprétation des données, à la critique du contenu et à la rédaction du manuscrit.

Ce travail a été élaboré dans le cadre d'un travail académique (mémoire pour le Diplôme inter-universitaire de pédagogie médicale, Universités d'Angers, Brest, Nantes et Rennes, 2015-2016).

\section{Conflits d'intérêts}

Les auteurs déclarent n'avoir aucun lien d'intérêt concernant les données publiées dans cet article.

\section{Approbation éthique}

Non sollicitée.

\section{Références}

1. Topping KJ. The effectiveness of peer tutoring in further and higher education: a typology and review of the literature. High Educ 1996;32:321-45.

2. Burgess A, McGregor D, Mellis C. Medical students as peer tutors: a systematic review. BMC Med Educ 2014;14:115.

3. Pålsson Y, Mårtensson G, Swenne CL, Ädel E, Engström M. A peer learning intervention for nursing students in clinical practice education: a quasi-experimental study. Nurse Educ Today 2017;51:81-87.

4. Parent F, Jouquan J. Comment élaborer et analyser un référentiel de compétences en santé? Louvain la Neuve : De Boeck Supérieur, 2015, p. 237-245.
5. Arrêté du 5 juillet 2012 relatif au diplôme d'État de pédicure-podologue. Bulletin Officiel Santé - Protection sociale - Solidarité 2012/6 du 15 juillet 2012:219-340.

6. Tardif J. Quels sont les grands principes et actions pédagogiques associés aux principales théories de l'apprentissage, in Comment (mieux) former et évaluer les étudiants en médecine et en sciences de la santé?, Pelaccia T. De Boeck Supérieur : Louvain la Neuve, 2016, p. 153-157.

7. Paul M. L'accompagnement: une nébuleuse. Rech Form 2009;62:91-108.

8. Menaut H. Tutorat et formations paramédicales. Penser l'accompagnement tutoral. Bruxelles : De Boeck, 2013.

9. Legendre R. Taxonomie, in Dictionnaire actuel de l'éducation, Legendre R. Montréal (Québec) : Guérin (3éd.), 2005, p. 1320-1322.

10. Mercier K, Pruneau H. L'apprentissage par les pairs : une solution gagnante pour les stages en soins infirmiers. Actes du $30^{\mathrm{e}}$ colloque de l'AQPC (Sherbrooke). Montréal : AQPC, 2010;508:115-119.

11. Loke AJ, Chow FL. Learning partnership-The experience of peer tutoring among nursing students: a qualitative study. Int J Nurs Stud 2007;44:237-44.

12. Bulte C, Betts A, Garner K, Durning S. Student teaching: views of student near-peer teachers and learners. Med Teach 2007;29:583-90.

13. Chamberland M, Hivon R. Les compétences de l'enseignant clinicien et le modèle de rôle en formation clinique. Pédagogie Médicale 2005;6:98-111.

14. Albero B. De l'idéel au vécu: le dispositif confronté à ses pratiques, in Enjeux et dilemmes de l'autonomie. Une expérience d'autoformation à l'université. Étude de cas, Albero B, Poteaux N. Maison des Sciences de l'Homme: Paris, 2010, p. 67-94.

15. Demeuse M. Échelles de Likert ou méthode des classements additionnés, in Introduction aux théories et aux méthodes de la mesure en sciences psychologiques et en sciences de l'éducation. Notes de cours. (Partie V, chapitre 3), Demeuse M. Institut de Recherche sur l'Éducation : Master Sociologie et Économie de l'Éducation Sciences de l'éducation Expertise Sociologique et Économique en éducation (ESE) 2008 [En ligne] Disponible sur: http://iredu.u-bourgogne. $\mathrm{fr} /$ images/stories/Documents/Cours_disponibles/ Demeuse/Cours/racine.pdf.

16. Carifio J, Perla R. Resolving the 50-year debate around using and misusing Likert scales. Med Educ 2008;42:11501152.

17. Foray P. Autonomie. Le Télémaque 2017;51;19-28.

Citation de l'article : Delahaye-Muller K., Le Faou Y., Chales G., Apprentissage en binôme collaboratif dans l'enseignement pratique de la formation initiale des pédicures-podologues : une enquête de terrain. Pédagogie Médicale 2017:18;199-205 